TRANSACTIONS OF THE

AMERICAN MATHEMATICAL SOCIETY

Volume 348, Number 12, December 1996, Pages 5065-5085

S $0002-9947(96) 01858-2$

\title{
INTEGRAL TYPE LINEAR FUNCTIONALS ON ORDERED CONES
}

\author{
WALTER ROTH
}

\begin{abstract}
We introduce linear functionals on an ordered cone that are minimal with respect to a given subcone. Using concepts developed for Choquet theory we observe that the properties of these functionals resemble those of positive Radon measures on locally compact spaces. Other applications include monotone functionals on cones of convex sets, H-integrals on $\mathrm{H}$-cones in abstract potential theory, and classical Choquet theory itself.
\end{abstract}

\section{INTRODUCTION}

The general theory of locally convex cones as developed in [7] deals with ordered cones that are not necessarily embeddable in vector spaces. A topological structure is introduced using order theoretical concepts. Staying reasonably close to the theory of locally convex spaces, this approach yields a sufficiently rich duality theory including Hahn-Banach type extension and separation theorems for linear functionals. We shall review some of the main concepts. We globally refer to [7] for details and proofs:

An ordered cone (cf. [6] and [7]) is a set $P$ endowed with an addition $(a, b) \mapsto a+b$ and a scalar multiplication $(\alpha, a) \mapsto \alpha a$ for real numbers $\alpha \geq 0$. The addition is supposed to be associative and commutative, and there is a neutral element $0 \in P$. For the scalar multiplication the usual associative and distributive properties hold, i.e. $\alpha(\beta a)=(\alpha \beta) a,(\alpha+\beta) a=\alpha a+\beta a$ and $\alpha(a+b)=\alpha a+\beta a$ for all $a, b \in P$ and $\alpha, \beta \geq 0$. We have $1 a=a$ and $0 a=0$ for all $a \in P$. The cancellation law, stating that $a+c=b+c$ implies $a=b$, however, is not required in general. It holds if and only if the cone $P$ may be embedded into a real vector space. Also, $P$ carries a (partial) order, i.e. a reflexive transitive relation $\leq$ such that $a \leq b$ implies $a+c \leq b+c$ and $\alpha a \leq \alpha b$ for all $a, b, c \in P$ and $\alpha \geq 0$.

A linear functional on a cone $P$ is a mapping $\mu: P \rightarrow \overline{\mathbb{R}}=\mathbb{R} \cup\{+\infty\}$ such that $\mu(a+b)=\mu(a)+\mu(b)$ and $\mu(\alpha a)=\alpha \mu(a)$ for all $a, b \in P$ and $\alpha \geq 0$. In $\overline{\mathbb{R}}$ we consider the usual algebraic operations, in particular $\alpha+\infty=+\infty$ for all $\alpha \in \overline{\mathbb{R}}$, $\alpha \cdot(+\infty)=+\infty$ for all $\alpha>0$ and $0 \cdot(+\infty)=0$. The functional $\mu$ is called monotone if $a \leq b$ implies $\mu(a) \leq \mu(b)$. Note that linear functionals can assume only finite values in elements $a \in P$ such that $-a \in P$ as well.

An ordered cone $P$ is called a full locally convex cone if it contains an abstract neighborhood system $V$, i.e. a subset of positive elements that is directed downward,

Received by the editors June 27, 1994.

1991 Mathematics Subject Classification. Primary 46A55, 47H05.

Key words and phrases. Locally convex cones, abstract integrals.

(C)1996 American Mathematical Society 
closed for addition and multiplication by (strictly) positive scalars. The elements $v$ of $V$ define upper resp. lower neighborhoods for the elements of $P$ by

$$
v(a)=\{b \in P \mid b \leq a+v\} \quad \text { resp. } a(v)=\{b \in P \mid a \leq b+v\},
$$

creating the upper resp. lower topologies on $P$. Their common refinement is called the symmetric topology. All elements of $P$ are supposed to be bounded below, i.e. for every $a \in P$ and $v \in V$ we have $0 \leq a+\rho v$ for some $\rho>0$. Finally, a locally convex cone is a subcone of a full locally convex cone not necessarily containing the abstract neighborhood system $V$. Every locally convex ordered topological vector space is a locally convex cone in this sense, as it may be canonically embedded into a full locally convex cone (cf. [7], Example I.2.7).

The polar $v_{P}^{\circ}$ of a neighborhood $v \in V$ consists of all linear functionals $\mu$ on $P$ satisfying $\mu(a) \leq \mu(b)+1$, whenever $a \leq b+v$ for $a, b \in P$. A linear functional belonging to the polar of some neighborhood is said to be (uniformly) continuous. Continuity requires that $\mu$ is monotone, and for a full cone $P$ it means just that $\mu(v) \leq 1$ holds for some $v \in V$ in addition. The Extension Theorem II.2.9 from [7] states that for a subcone $Q$ of $P$, every linear functional in $v_{Q}^{\circ}$ extends to an element of $v_{P}^{\circ}$. The continuous linear functionals on a locally convex cone $P$ form again a cone, called the dual cone of $P$ and denoted by $P^{*}$. We endow $P^{*}$ with the topology $w\left(P^{*}, P\right)$ of pointwise convergence of the elements of $P$, considered as functions on $P^{*}$ with values in $\overline{\mathbb{R}}$ with its usual topology. The polar $v_{P}^{\circ}$ of a neighborhood $v \in V$ is seen to be $w\left(P^{*}, P\right)$-compact and convex ([7], Theorem II.2.4).

For the sake of simplicity, we shall develop the concept of integral type linear functionals for full locally convex cones only. Our theory applies, however, to locally convex cones in general, because every such cone may (by definition) be embedded into a full cone. Throughout this paper we shall use the following examples of full locally convex cones to illustrate our concepts:

Examples 1.1. (a) Let $X$ be a compact convex subset of a locally convex Hausdorff space, and let $P$ be the set of all bounded below $\overline{\mathbb{R}}$-valued functions on $X$, endowed with the usual algebraic operations and the pointwise order. Let the abstract neighborhood system $V$ consist of all strictly positive lower semicontinuous concave functions on $X$. Clearly $V$ is directed downward (the pointwise infimum of two functions in $V$ is again in $V$ ) and closed for addition and multiplication by positive scalars. Every function in $V$ attains its strictly positive minimum value on $X$, and as the functions in $P$ are bounded below, for every $f \in P$ and $v \in V$ there is $\rho>0$ such that $0 \leq f+\rho v$, indeed. Accordingly $P$ is a full locally convex cone.

(b) Let $P$ be the cone of all non-empty closed convex subsets of a locally convex vector space $E$, endowed with the usual multiplication by scalars of sets, a slightly modified addition $\oplus$, where $\mathcal{A} \oplus \mathcal{B}$ denotes the closure of the usual sum of the sets $\mathcal{A}$ and $\mathcal{B}$, and the set inclusion as the canonical order. The abstract neighborhood system in $P$ is given by a basis $V \subset P$ of closed absolutely convex neighborhoods of the origin in $E$. For every $\mathcal{A} \in P$ and $\mathcal{V} \in V$ there is $\rho>0$ such that $\rho \mathcal{V} \cap \mathcal{A} \neq \emptyset$, hence $\{0\} \subset \mathcal{A}+\rho \mathcal{V}$. Thus every element of $P$ is indeed bounded below, and $P$ is a full locally convex cone.

Via the embedding of its elements into singleton subsets, the space $E$ itself may be considered as a subcone of $P$. On this subcone the three (upper, lower and symmetric) topologies of $P$ coincide with the given topology on $E$. Thus $E$ is a locally convex cone, but not a full cone. Other subcones of $P$ that merit further 
investigation are those of all bounded, resp. all compact sets in $P$. They satisfy the cancellation law. Details on those and further related examples may be found in [7], I.1.7, I.2.7 and I.2.8.

(c) The following example will model topological integration theory as presented in [2], [3] and [5]: Let $X$ be a locally compact Hausdorff space. By $F$ we denote the set of all $\overline{\mathbb{R}}$-valued functions on $X$ that are bounded below and assume negative values only on a relatively compact subset of $X$. We use the pointwise order and algebraic operations for the functions in $F$. A neighborhood (in the topology of the inductive limit) is a convex subset $v$ of lower semicontinuous functions in $F$ that contains a positive multiple of the characteristic function of every relatively compact open subset of $X$. The set $V$ of all those neighborhoods $v$ is directed downward and closed for the usual addition of sets and multiplication by positive scalars. But the neighborhood system $V$ is obviously not contained in the cone $F$, thus in order to create a full locally convex cone we have to adjoin the elements of $V$ using a procedure that is elaborated in a more general context in [7], Ch. I.5:

Let $P$ consist of all objects $f \oplus v$, for $f \in F$ and $v \in V \cup\{\{0\}\}$. We define the algebraic operations by

$$
(f \oplus v)+\left(f^{\prime} \oplus v^{\prime}\right)=\left(f+f^{\prime}\right) \oplus\left(v+v^{\prime}\right) \quad \text { and } \quad \alpha(f \oplus v)=(\alpha f \oplus \alpha v),
$$

and the order by: $f \oplus v \leq f^{\prime} \oplus v^{\prime}$ if for every $h \in v$ there is $h^{\prime} \in v^{\prime}$ such that $f+h \leq f^{\prime}+h^{\prime}$. Via the embedding $v \mapsto 0 \oplus v$, the neighborhood system $V$ may be considered as a subset of $P$. It is straightforward to check that every element in $P$ is bounded below as required: Given an element $f \oplus w \in P$ and a neighborhood $0 \oplus v$, by the definition of $F$ the function $f$ is bounded below and attains negative values only on a relatively compact subset $Y$ of $X$. Consequently we find a relatively compact open set $Z \supset Y$ and a constant $\kappa>0$ such that $f \geq-\kappa \chi_{z}$, where $\chi_{Z}$ denotes the (lower semicontinuous) characteristic function of $Z$. But $\varepsilon \chi_{Z} \in v$ for some $\varepsilon>0$. Set $\rho=\kappa / \varepsilon$. Then $0 \leq f+\rho\left(\varepsilon \chi_{z}\right)$, hence

$$
0 \oplus\{0\} \leq f \oplus(w+\rho v)=(f \oplus w)+(0 \oplus \rho v),
$$

indeed. Thus, $P$ becomes a full locally convex cone. As $F$ itself, via the embedding $f \mapsto f \oplus\{0\}$, is a subcone of $P$, we observe that $F$ is a locally convex cone, but not a full cone.

This example simplifies considerably if we restrict ourselves to compact Hausdorff spaces $X$. In this case, for the neighborhood system $V$ we may choose the subset of all strictly positive lower semicontinuous functions on $X$. Then $V \subset F$, and $F$ is already a full cone. The extension procedure for the construction of $P$ from the general case is therefore superfluous.

(d) An $H$-cone $P$, as introduced in abstract potential theory (for details cf. [4]) is an ordered cone of positive elements. The cancellation law holds as well as the Riesz decomposition property; i.e. for all $a, b, c \in P$ such that $a \leq b+c$ there are $b^{\prime}, c^{\prime} \in P$ such that $a=b^{\prime}+c^{\prime}$ and $b^{\prime} \leq b, c^{\prime} \leq c$. Furthermore, in an H-cone $P$, for every dominated and directed upward family $F \subset P$ there exists the supremum $\bigvee F$, and $a+\bigvee F=\bigvee(a+F)$ holds for all $a \in P$. Likewise, for every non-empty family $F \subset P$ the infimum $\bigwedge F$ exists, and $a+\bigwedge F=\bigwedge(a+F)$ holds for all $a \in P$. A weak unit is an element $v \in P$ such that $a=\bigvee_{n \in \mathbb{N}}(a \wedge n v)$ for all $a \in P$. For our purposes, we request that weak units do exist in $P$. (This is, for example, guaranteed for a standard $\mathrm{H}$-cone $P$ as defined in [4]). Let the abstract neighborhood system $V \subset P$ consist of all such weak units. As shown in [4], 4.1, the set $V$ is directed 
downward and closed for addition and multiplication by positive scalars, thus fulfils the requirements for an abstract neighborhood system. As all the elements of $P$ are positive, they are obviously bounded below.

\section{Minimal Linear FunCtionals on a LOCALly CONVEX CONE}

In the following, let $P$ be a full locally convex cone with the abstract neighborhood system $V \subset P$. Let $R$ be a subcone of $P$ that also contains $V$; i.e. $R$ itself is a full locally convex cone. We shall use this subcone to define an order relation $\preccurlyeq R$ for linear functionals $\mu$ and $\nu$ in the dual cone $P^{*}$ of $P$ as follows:

$$
\mu \preccurlyeq R \nu \quad \text { if and only if } \quad \mu(r) \leq \nu(r) \quad \text { for all } \quad r \in R .
$$

Using concepts from classical Choquet theory (cf. [1] and [8]), we shall investigate minimal elements with respect to this order on $P^{*}$. Two linear functionals $\mu$ and $\nu$ in $P^{*}$ are called $R$-equivalent if they coincide on the subcone $R$. We shall write $\mu \widetilde{\widetilde{R}} \nu$. The functional $\mu \in P$ is called $R$-minimal if $\nu \preccurlyeq_{R} \mu$ implies that $\mu \widetilde{R}_{\tilde{R}} \nu$ for every $\nu \in P^{*}$.

Theorem 2.1. For every linear functional $\mu_{0} \in P^{*}$ there is an $R$-minimal functional $\mu \in P^{*}$ such that $\mu \preccurlyeq_{R} \mu_{0}$.

Proof. First we note that for every $\nu \in P^{*}$, the set $Z_{\nu}=\left\{\rho \in P^{*} \mid \rho \preccurlyeq R \quad \nu\right\}$ is $w\left(P^{*}, P\right)$-compact: Indeed, $Z_{\nu}$ is contained in the compact polar of a neighborhood (recall that $\nu(v) \leq 1$ for some $v \in V \subset R$, and that $\rho \preccurlyeq R \nu$ implies $\rho(v) \leq \nu(v)$ ), and $Z_{\nu}$ is closed in this polar as $Z_{\nu}=\bigcap_{r \in R}\left\{\rho \in P^{*} \mid \rho(r) \leq \nu(r)\right\}$. Thus, given a chain $T$ in $Z_{\mu_{0}}$, the intersection of all $Z_{\nu}, \nu \in T$, cannot be empty, hence contains a lower bound for $T$ in $Z_{\mu_{0}}$. By Zorn's Lemma there is a minimal element $\mu$ in $Z_{\mu_{0}}$.

As in Choquet theory we use envelopes to characterize $R$-minimal linear functionals:

Definition 2.2. For $a \in P$ and $\mu \in P^{*}$, set

$$
\begin{aligned}
& \check{\mu}(a)=\sup \{-\mu(r) \mid 0 \leq a+r, r \in R\} \quad \text { and } \\
& \hat{\mu}(a)=\inf \{\mu(r) \mid a \leq r, r \in R\} .
\end{aligned}
$$

We observe the following properties:

Lemma 2.3. Let $a \in P$ and $\mu, \nu \in P^{*}$.

(a) $-\infty<\check{\mu}(a) \leq \mu(a) \leq \hat{\mu}(a)$.

(b) If $\nu \preccurlyeq_{R} \mu$, then $\check{\mu}(a) \leq \check{\nu}(a)$ and $\hat{\nu}(a) \leq \hat{\mu}(a)$.

Proof. For part (a) we recall that $\mu(v) \leq 1$ holds for some $v \in V \subset R$ and $0 \leq$ $a+\rho v$ for some $\rho>0$, as all elements of the locally convex cone $P$ are required to be bounded below. Consequently, $\breve{\mu}(a) \geq-\mu(\rho v) \geq-\rho>-\infty$. Furthermore, $0 \leq a+r$ for some $r \in R$ implies that $0 \leq \mu(a)+\mu(r)$, whence $-\mu(r) \leq \mu(a)$. Thus $\check{\mu}(a) \leq \mu(a)$, indeed. As $\mu$ is monotone, $\mu(a) \leq \hat{\mu}(a)$ is obvious. Part (b) of the lemma follows straight from Definition 2.2.

Lemma 2.4. Let $a \in P$ and $\mu \in P^{*}$.

(a) There is $\nu \in P^{*}$ such that $\nu \preccurlyeq_{R} \mu$ and $\nu(a)=\check{\mu}(a)$.

(b) There is $\nu \in P^{*}$ such that $\nu \preccurlyeq_{R} \mu$ and $\nu(a)=\hat{\mu}(a)$. 
Proof. Let $\mu \in P^{*}$ and $a \in P$. For part (a) of the lemma set

$$
p(b)=\inf \{\mu(r)+\lambda \check{\mu}(a) \mid b \leq r+\lambda a \quad \text { for } \quad r \in R, \lambda \geq 0\} \quad \text { for } \quad b \in P .
$$

First we have to verify that $p(b)>-\infty$ for all $b \in P$ : There is a neighborhood $v \in V$ such that $\mu(v) \leq 1$, and for $b \in P$ there is $\rho>0$ such that $0 \leq b+\rho v$. Now let $r \in R$ and $\lambda \geq 0$ such that $b \leq r+\lambda a$. The combination of both inequalities for $b$ yields that $0 \leq r+\lambda a+\rho v=\lambda a+(r+\rho v)$. As $r+\rho v \in R$, the latter shows by the definition of the envelope that $-\mu(r+\rho v) \leq \check{\mu}(\lambda a)=\lambda \check{\mu}(a)$, i.e. $\mu(r)+\lambda \check{\mu}(a) \geq-\mu(\rho v) \geq-\rho$. Thus we have demonstrated that $p(b) \geq-\rho>-\infty$, indeed.

It is straightforward to check that $p$ is sublinear and monotone on $P$. As obviously $p(r) \leq \mu(r)$ holds for all $r \in R$, we have $p(v) \leq \mu(v) \leq 1$ for the neighborhood $v$ from above, and $p$ is seen to be $u$-continuous (cf. [7], II.2.5). Now we are ready to apply the Sandwich Theorem II.2.8 from [7] for $p$ (and the superlinear functional $q(b)=-\infty$ for all $0 \neq b \in P)$ : There is a linear functional $\nu \in P^{*}$ such that $\nu(b) \leq p(b)$ for all $b \in P$. We check very easily that this functional $\nu$ fulfils the statement of our lemma:

We have $\nu(r) \leq p(r) \leq \mu(r)$ for all $r \in R$, hence $\nu \preccurlyeq_{R} \mu$. Clearly, $\nu(a) \leq$ $p(a) \leq \check{\mu}(a)$ for the fixed element $a \in P$. But on the other hand, $\nu \preccurlyeq_{R} \mu$ implies by Lemma $2.3(\mathrm{~b})$ that $\check{\mu}(a) \leq \nu(a)$ holds as well, thus completing our proof of part (a).

For part (b) of the lemma we set

$$
p(b)=\hat{\mu}(b)=\inf \{\mu(r) \mid b \leq r, r \in R\} \quad \text { for } \quad b \in P .
$$

Clearly $p$ is sublinear, monotone and $u$-continuous, as $p(v) \leq \mu(v) \leq 1$ for the same neighborhood $v$ as above. We define a superlinear functional $q$ on $P$ with values in $\mathbb{R} \cup\{-\infty,+\infty\}$ by $q(b)=p(b)$ if $b=\lambda a$ for some $\lambda \geq 0$, and $q(b)=-\infty$ else. Then $q(b) \leq p(b)$ for all $b \in P$, and by the Sandwich Theorem there is $\nu \in P^{*}$ such that $q(b) \leq \nu(b) \leq p(b)$ for all $b \in P$. This shows in particular that $\nu(r) \leq p(r)=\mu(r)$ for all $r \in R$, i.e. $\nu \preccurlyeq R \mu$, and $q(a)=\nu(a)=p(a)=\hat{\mu}(a)$.

Theorem 2.5. For a linear functional $\mu \in P^{*}$ the following statements are equivalent:

(a) $\mu$ is R-minimal.

(b) $\check{\mu}(a)=\inf \left\{\nu(a) \mid \nu \in P^{*}, \nu \widetilde{R} \mu\right\}$ for all $a \in P$ and $\hat{\mu}(a)=\sup \left\{\nu(a) \mid \nu \in P^{*}, \nu \widetilde{R}^{\mu}\right\}$ for all $a \in P$

(c) $\check{\mu}(r)=\mu(r)$ for all $r \in R$.

Proof. In order to show that (a) implies (b), let $\mu \in P^{*}$ be $R$-minimal, and let $a \in P$. By part (a) of the preceding lemma there is $\nu \preccurlyeq_{R} \mu$, i.e. $\nu \widetilde{R} \mu$ by the minimality of $\mu$, such that $\nu(a)=\check{\mu}(a)$. On the other hand, if $\nu \widetilde{R} \mu$, i.e. $\nu \preccurlyeq_{R} \mu$, then $\check{\mu}(a) \leq \nu(a)$ by Lemma 2.3(b). Similarly, by part (b) of the lemma there is $\nu_{\widetilde{R}} \mu$ such that $\nu(a)=\hat{\mu}(a)$. As $\nu(a) \leq \hat{\nu}(a)=\hat{\mu}(a)$ holds for any $\nu_{\widetilde{R}} \mu$, the second equality in (b) is also verified.

Obviously (b) implies (c), because, $\nu \widetilde{R}_{\mu} \mu$ means that $\nu(r)=\mu(r)$ holds for all $r \in R$.

Finally, if (c) holds for $\mu$ and $\nu \preccurlyeq R \mu$ for some $\nu \in P^{*}$, then for all $r \in R$ we conclude using Lemma 2.3(b) that $\mu(r)=\check{\mu}(r) \leq \nu(r)$, whence $\mu \preccurlyeq_{R} \nu$ as well, and $\mu$ is seen to be $R$-minimal, indeed. 


\section{INTEGRALS}

We shall use the previously developed concepts in order to define integral type linear functionals on a full locally convex cone $P$. First let us recall that an element $b$ of a locally convex cone $P$ is called bounded (above) (cf. [7], I.2.3) if for every abstract neighborhood $v \in V$ there is $\rho>0$ such that $b \leq \rho v$. For bounded elements it may be shown that a modified version of the cancellation law holds ([7], Lemma I.4.3): For $a, c \in P$ and a bounded element $b \in P, a+b \leq c+b$ implies that $a \leq c+v$ for all $v \in V$. We denote by $B$ the subcone of bounded elements of $P$. Continuous linear functionals assume only finite values on $B$.

A locally convex cone $P$ does not need to be separated in the (upper resp. lower) topologies induced by the respective neighborhoods. But $P$ may be projected onto a separated locally convex cone $\bar{P}$ that reflects all the essential properties of $P$. Moreover, $\bar{P}$ may be extended to contain $-\bar{B}$, the negatives of the bounded elements of $P$. For details of this procedure we refer to [7], I.3 and I.4. Moreover, the duals of $P$ and $\bar{P}-\bar{B}$ may be identified ([7], Proposition II.2.2).

To establish our theory we select two subcones $L$ and $U$ of $P$. $L$ is supposed to be a full cone, whereas all elements of $U$ are supposed to be bounded. We require that the following two conditions hold:

(U) For all $a \in P, l \in L, u \in U$ such that $u \leq a+l$ and for every $v \in V$ there is $u^{\prime} \in U$ such that $u^{\prime} \leq a+v$ and $u \leq u^{\prime}+l+v$.

(L) For all $a \in P, l \in L, u \in U$ such that $a+u \leq l$ and for every $v \in V$ there is $l^{\prime} \in L$ such that $a \leq l^{\prime}$ and $l^{\prime}+u \leq l+v$.

We shall give suitable choices for the subcones $L$ and $U$ in our Examples 1.1(a) to (d) at the end of this section.

We are now ready to apply the concept of of $R$-minimal functionals as developed in Section 1. However, we shall use the full locally convex cone $\bar{P}-\bar{B}$ instead of $P$ and the full subcone $R=\bar{L}-\bar{U}$ to define the order relation $\preccurlyeq_{R}$ on $(\bar{P}-\bar{B})^{*}$ which by the above remark may be identified with $P^{*}$. For linear functionals $\mu, \nu \in P^{*}$ now we have

$$
\mu \preccurlyeq_{R} \nu \text { if } \mu(l) \leq \nu(l) \text { for all } l \in L \text { and } \mu(u) \geq \nu(u) \text { for all } u \in U .
$$

We shall call integrals on $P$ the minimal functionals in this order and refer to $(P, L, U)$ as an integration cone.

The envelopes as defined in 2.2 may now be expressed as follows:

Lemma 3.1. For $a \in P$ and $\mu \in P^{*}$ we have

$$
\begin{aligned}
& \check{\mu}(a)=\inf _{v \in V} \sup \{\mu(u) \mid u \leq a+v, u \in U\} \quad \text { and } \\
& \hat{\mu}(a)=\inf \{\mu(l) \mid a \leq l, l \in L\} .
\end{aligned}
$$

Proof. For $\check{\mu}(a)$ we argue as follows: Let $\varepsilon>0$ and let $v \in V$ such that $\mu(v) \leq \varepsilon$. For $u \in U$ such that $u \leq a+v$ we have $0 \leq a+(v-u)$ and $\check{\mu}(a) \geq-\mu(v-u) \geq \mu(u)-\varepsilon$ by Definition 2.2, as $(v-u) \in R$. Consequently

$$
\check{\mu}(a) \geq \inf _{v \in V} \sup \{\mu(u) \mid u \leq a+v, u \in U\}
$$

holds.

On the other hand, let $(l-u) \in R$ such that $0 \leq a+(l-u)$, i.e. $u \leq a+l$. For $\varepsilon>0$ and any $v \in V$ such that $\mu(v) \leq \varepsilon$, by condition (U) there is $u^{\prime} \in U$ such that $u^{\prime} \leq a+v$ and $u \leq u^{\prime}+l+v$, whence $\mu(u) \leq \mu\left(u^{\prime}\right)+\mu(l)+\varepsilon$, i.e. $-\mu(l-u) \leq \mu\left(u^{\prime}\right)+\varepsilon$. We infer that $\check{\mu}(a) \leq \sup \{\mu(u) \mid u \leq a+v, u \in U\}+\varepsilon$. Because the latter 
inequality holds for all $v \in V$ and because $V$ is directed downward, we have even $\check{\mu}(a) \leq \inf _{v \in V} \sup \{\mu(u) \mid u \leq a+v, u \in U\}+\varepsilon$. As $\varepsilon>0$ was arbitrary, this verifies our claim for $\check{\mu}(a)$.

Similarly, in order to establish the expression for $\hat{\mu}(a)$, we observe immediately from Definition 2.2 that $\hat{\mu}(a) \leq \inf \{\mu(l) \mid a \leq l, l \in L\}$ holds, as $L \subset R$. On the other hand, let $a \leq l-u$ for some $l-u \in R$, i.e. $a+u \leq l$. For $\varepsilon>0$ and $v \in V$ such that $\mu(v) \leq \varepsilon$ by (L) there is $l^{\prime} \in L$ such that $a \leq l^{\prime}$ and $l^{\prime}+u \leq l+v$, i.e. $\mu\left(l^{\prime}\right) \leq \mu(l-u)+\varepsilon$. The latter demonstrates that $\inf \{\mu(l) \mid a \leq l, l \in L\} \leq \hat{\mu}(a)+\varepsilon$, hence the reverse inequality holds as well.

Theorem 3.2. The linear functional $\mu \in P^{*}$ is an integral if and only if $\check{\mu}(l)=\mu(l)$ for all $l \in L$ and $\hat{\mu}(u)=\mu(u)$ for all $u \in U$.

Proof. The necessity of this condition for an integral $\mu$ is clear from Theorem 2.5. Suppose on the other hand that the condition holds for $\mu \in P^{*}$ and let $\nu \in P^{*}$ such that $\nu \preccurlyeq_{R} \mu$. By Lemma 2.3(b), for all $l \in L$ and all $u \in U$ we have $\mu(l)=\check{\mu}(l) \leq$ $\check{\nu}(l) \leq \nu(l)$ and $\mu(u)=\hat{\mu}(u) \geq \hat{\nu}(u) \geq \nu(u)$, Thus, $\mu \preccurlyeq R \nu$ holds as well, and $\mu$ is seen to be $R$-minimal.

The following is a straightforward transcription of Theorem 2.1. Recall that every continuous linear functional on a subcone of a locally convex cone may be extended to the whole cone. Moreover, if the functional is contained in the polar of a certain neighborhood relative to this subcone, its extension may be found in the polar of the same neighborhood relative to the whole cone (cf. [7], Theorem II.2.9).

Theorem 3.3. For every continuous linear functional $\mu_{0} \in P^{*}$ there is an integral $\mu$ on $P$ such that

$$
\mu(l) \leq \mu_{0}(l) \quad \text { for all } \quad l \in L \quad \text { and } \quad \mu(u) \geq \mu_{0}(u) \text { for all } u \in U .
$$

We proceed to define integrability with respect to an integral on $P$.

Definition 3.4. Let $(P, L, U)$ be an integration cone and let $\mu$ be an integral on $P$. The element $a \in P$ is said to be $\mu$-integrable if $\nu \widetilde{R}_{\mu} \mu$ implies that $\nu(a)=\mu(a)$ for all $\nu \in P^{*}$.

Note that for a given integral $\mu$ on $P$ the $\mu$-integrable elements form a subcone of $P$ that contains $L+U$.

Lemma 3.5. Let $a \in P$ and $v \in V$. If for every $\varepsilon>0$ there are $b_{1}, b_{2} \in L+U$ such that $b_{2}$ is bounded and $b_{1} \leq a+b_{2} \leq b_{1}+\varepsilon v$, then the element a is $\mu$-integrable for every integral $\mu$ on $P$ such that $\mu(v)<+\infty$.

Proof. Let $\mu$ be an integral such that $\mu(v)<+\infty$ and let $\nu \widetilde{R}_{\mu}$. For $\varepsilon>0$ let $b_{1}, b_{2} \in L+U$ be as in the lemma. The functionals $\mu$ and $\nu$ coincide on $b_{1}$ and $b_{2}$ and on $v$, and

$$
\mu\left(b_{1}\right) \leq \mu(a)+\mu\left(b_{2}\right) \leq \mu\left(b_{1}\right)+\varepsilon \mu(v)
$$

as well as

$$
\nu\left(b_{1}\right) \leq \nu(a)+\nu\left(b_{2}\right) \leq \nu\left(b_{1}\right)+\varepsilon \nu(v) .
$$

As $b_{2}$ is bounded, we know that $\mu\left(b_{2}\right)=\nu\left(b_{2}\right)<+\infty$. If $\mu\left(b_{1}\right)=\nu\left(b_{1}\right)=+\infty$, then $\mu(a)=\nu(a)=+\infty$ as well. Otherwise we have

$$
\left|\mu(a)+\mu\left(b_{2}\right)-\mu\left(b_{1}\right)\right| \leq \varepsilon \mu(v) \quad \text { and } \quad\left|\nu(a)+\nu\left(b_{2}\right)-\nu\left(b_{1}\right)\right| \leq \varepsilon \nu(v),
$$


whence $|\mu(a)-\nu(a)| \leq \varepsilon(\mu(v)+\nu(v))$. This holds for every $\varepsilon>0$ and shows that $\mu(a)=\nu(a)$, indeed.

Theorem 3.6. Let $\mu$ be an integral on $P$. The element $a \in P$ is $\mu$-integrable if and only if

$$
\begin{aligned}
\mu(a) & =\inf _{v \in V} \sup \{\mu(u) \mid u \leq a+v, u \in U\} \\
& =\inf \{\mu(l) \mid a \leq l, l \in L\} .
\end{aligned}
$$

Proof. If $a \in P$ is $\mu$-integrable, then the statement follows directly from Theorem 2.5 and Lemma 3.1. If on the other hand the above equality holds for $a$ and if $\nu \widetilde{R} \mu$, then we conclude that

$$
\nu(a) \leq \hat{\nu}(a)=\hat{\mu}(a)=\mu(a)=\check{\mu}(a)=\check{\nu}(a) \leq \nu(a),
$$

i.e. $\nu(a)=\mu(a)$, indeed.

We shall say that two integrals $\mu$ and $\nu$ on $P$ are equivalent and denote $\mu \equiv \nu$, if they generate the same integrable elements in $P$ and coincide on them. Theorem 3.6 shows that any two integrals that coincide on $U$ or on $L$ are already equivalent.

Definition 3.7. The integration cone $(P, L, U)$ is called simplicial if for all $u \in U$ and $l \in L$ such that $u \leq l$ there is $c \in C=U \cap L$ such that $u \leq c \leq l$.

Note that any integration cone $(P, L, U)$ such that $U \subset L$ is obviously simplicial.

Theorem 3.8. If $(P, L, U)$ is simplicial, then

$$
\begin{aligned}
& \mu(l)=\inf _{v \in V} \sup \{\mu(c) \mid c \leq l+v, c \in C\} \quad \text { and } \\
& \mu(u)=\inf \{\mu(c) \mid u \leq c, c \in C\}
\end{aligned}
$$

for all $l \in L, u \in U$ and every integral $\mu$ on $P$.

Proof. Let $\mu$ be an integral on $P$. For every $l \in L$ we have by 3.6 that

$$
\mu(l)=\inf _{v \in V} \sup \{\mu(u) \mid u \leq l+v, u \in U\} \geq \inf _{v \in V} \sup \{\mu(c) \mid c \leq l+v, c \in C\} .
$$

On the other hand, let $v \in V$ and $u \in U$ such that $u \leq l+v$. As $(P, L, U)$ is simplicial and as $l+v \in L$, there is $c \in C$ such that $u \leq c \leq l+v$, i.e. $\mu(u) \leq \mu(c)$. This demonstrates

$$
\begin{aligned}
\sup \{\mu(u) \mid u \leq l+v, u \in U\} & \leq \sup \{\mu(c) \mid c \leq l+v, c \in C\}, \quad \text { and } \\
\inf _{v \in V} \sup \{\mu(u) \mid u \leq l+v, u \in U\} & \leq \inf _{v \in V} \sup \{\mu(c) \mid c \leq l+v, \quad c \in C\} .
\end{aligned}
$$

Similarly, for $u \in U$ we see again by 3.6 that

$$
\mu(u)=\inf \{\mu(l) \mid u \leq l, l \in L\} \leq \inf \{\mu(c) \mid u \leq c, c \in C\} .
$$

But for any $l \in L$ such that $u \leq l$, by 3.6 there is an element $c \in C$ such that $u \leq c \leq l$. This shows that the reverse inequality holds as well.

Theorem 3.9. If the integration cone $(P, L, U)$ is simplicial, then for every $v \in V$ and every linear functional $\mu_{0} \in v_{C}^{\circ} \subset C^{*}$ there is a unique extension of $\mu_{0}$ which is an integral on $P$.

Proof. By the Extension Theorem II.2.9 from [7] for continuous linear functionals and our Theorem 3.3 there is an integral extension $\mu$ of $\mu_{0}$. The preceding lemma shows that the values of $\mu$ on $L$ and on $U$ are already determined by $\mu_{0}$. Thus any two integral extensions of $\mu_{0}$ are seen to be equivalent. 
Definition 3.10. The integration cone $(P, L, U)$ is said to be almost full, if for every $v \in V$ there is $v^{\prime} \in V$ such that for all $u \in U, a \in P$ satisfying $u \leq a+v^{\prime}$ there is $u^{\prime} \in U$ such that $u^{\prime} \leq a$ and $u \leq u^{\prime}+v$.

Note that the condition in Definition 3.10 is obviously fulfilled if $U$ contains $-V$. (Choose $v^{\prime}=v$ and $u^{\prime}=u-v$.) For almost full integration cones, the statements of Theorems 3.6 and 3.8 may be simplified using plain infima. The first part of our next claim will be immediate from the above definition. The second part follows from the first part if one repeats the argument of Theorem 3.8.

Theorem 3.11. If the integration cone $(P, L, U)$ is almost full, then

$$
\begin{aligned}
\mu(a) & =\sup \{\mu(u) \mid u \leq a, u \in U \\
& =\inf \{\mu(l) \mid a \leq l, l \in L\}
\end{aligned}
$$

holds for every integral $\mu$ on $P$ and every $\mu$-integrable element $a \in P$. If $(P, L, U)$ is almost full and simplicial, then

$$
\begin{aligned}
\mu(l) & =\sup \{\mu(c) \mid c \leq l, c \in C\} \quad \text { and } \\
\mu(u) & =\inf \{\mu(c) \mid u \leq c, c \in C\}
\end{aligned}
$$

holds for all $l \in L, u \in U$ and every integral $\mu$ on $P$.

Next we shall review our Examples 1.1 (a) to (d) and introduce suitable choices for the subcones $L$ and $U$. We shall start with some observations that allow to simplify our conditions for integration cones in special cases.

Remarks 3.12. (a) If $-U \subset L$, as in some of our following examples, then condition (L) holds with $l^{\prime}=l-u$, thus needs not to be checked. Combining the elements $l$ and $-u$, condition (U) may be simplified as follows:

$\left(\mathrm{U}^{\prime}\right) \quad$ For all $a \in P, l \in L$ such that $0 \leq a+l$ and for every $v \in V$ there is $u^{\prime} \in U$ such that $u^{\prime} \leq a+v$ and $0 \leq u^{\prime}+l+v$.

(b) If we have even $-U=L$, then condition (U) is also fulfilled: for any $v^{\prime} \in V$ choose $u^{\prime}=u-l$. $(P, L, U)$ is also an almost full cone in this case, as we may choose $v^{\prime}=v$ and $u^{\prime}=u-v$ in Definition 3.10, since $-V \subset-L=U$.

(c) If $L=P$, then condition (L) holds with $l^{\prime}=a$, thus needs not to be checked. Condition $(\mathrm{U})$ is seen to be equivalent to the following:

$\left(\mathrm{U}^{\prime \prime}\right)$ For all $a, b \in P$ and $u \in U$ such that $u \leq a+b$ and for every $v \in V$ there are $u^{\prime}, u^{\prime \prime} \in U$ such that $u^{\prime} \leq a+v, \quad u^{\prime \prime} \leq b+v$ and $u \leq u^{\prime}+u^{\prime \prime}+v$.

Examples 3.13. (a) In 1.1(a) we choose the subcone of all lower semicontinuous concave functions on $X$ for $L$ and the bounded upper semicontinuous convex functions for $U$. Then $V \subset L$ as required, and because $-U \subset L$, following Remark 3.12(a), we only have to verify condition $\left(\mathrm{U}^{\prime}\right)$ : Let $0 \leq f+l$ for $f \in P$ and $l \in L$. Let $\kappa \in \mathbb{R}$ be a lower bound for the function $f$ and set $u^{\prime}=\sup \{-l, \kappa\}$. Then $u^{\prime}$ is bounded, upper semicontiuous and convex, hence contained in $U$. Furthermore, we have $u^{\prime} \leq f$ and $0 \leq u^{\prime}+l$. Condition $\left(\mathrm{U}^{\prime}\right)$ therefore holds with any choice of a neighborhood $v \in V$.

Likewise, the integration cone $(P, L, U)$ is seen to be almost full since the condition in 3.10 holds with $v^{\prime}=v \in V$ : If $u \leq f+v$, i.e. $u-v \leq f$ for $f \in P$ and $u \in U$, choose $u^{\prime}=\sup \{u-v, \kappa\}$, where $\kappa \in \mathbb{R}$ denotes a lower bound for $f$. Then $u^{\prime} \in U$ and both $u^{\prime} \leq f$ and $u \leq u^{\prime}+v$. 
As the elements of the dual cone $P^{*}$ of $P$, when restricted to $C(X)$ are positive Radon measures on $X$, our integrals on $P$ are just the usual maximal representation measures from classical Choquet theory. It follows directly from Lemma 3.5 that the $\mu$-integrable elements of $P$ include all continuouus functions on $X$. (The closure of the subcone $L+U$ in $P$ obviously contains $C(X)$.)

Theorem 3.11 yields Mokobodzki's characterization (cf. [1], Proposition 1.4.5) of maximal measures in Choquet theory. The subcone $C=U \cap L$ consists of the continuous affine functions on $X$, and Theorem 3.3 states that every continuous (positive) linear functional on $C$ (i.e. a positive multiple of a point evaluation on $X)$ may be represented by such a maximal measure. This representation is unique if the integration cone $(P, L, U)$ is simplicial (Theorem 3.9), which in turn holds true if and only if the compact convex set $X$ is a Choquet simplex (cf. [1], Theorem II.3.10).

(b) In Example 1.2(b) we choose $L=P$ and for $U$ the subcone of $P$ of all singleton subsets of the locally convex space $E$. We shall check condition ( $\left.\mathrm{U}^{\prime \prime}\right)$ from 3.12 (c): Let $\mathcal{A}, \mathcal{B} \in P$ and $\mathcal{U}=\{u\} \in U$ such that $\mathcal{U} \leq \mathcal{A} \oplus \mathcal{B}$, i.e. $u \in \overline{\mathcal{A}+\mathcal{B}}$. Then for $\mathcal{V} \in V$ there are $a \in \mathcal{A}, b \in \mathcal{B}$ and $v \in \mathcal{V}$ such that $u=a+b+v$. We choose $u^{\prime}=a+v / 2$ and $u^{\prime \prime}=b+v / 2$. Then $u^{\prime} \in \mathcal{A}+\mathcal{V}$, i.e. $U^{\prime}=\left\{u^{\prime}\right\} \leq \mathcal{A} \oplus \mathcal{V}, u^{\prime \prime} \in \mathcal{B}+\mathcal{V}$, i.e. $U^{\prime \prime}=\left\{u^{\prime \prime}\right\} \leq \mathcal{B} \oplus \mathcal{V}$ and $u=u^{\prime}+u^{\prime \prime}$, i.e. $\mathcal{U}=\mathcal{U}^{\prime} \oplus \mathcal{U}^{\prime \prime}$, indeed.

As $U \subset L$, the integration cone $(P, L, U)$ is even simplicial, and also fulfils the criterion in Definition 3.10 for a full cone if we choose $\mathcal{V}^{\prime}=(1 / 2) \mathcal{V}$ : Let $\mathcal{U}=\{u\} \leq \mathcal{A} \oplus \mathcal{V}^{\prime} \subset \mathcal{A}+\mathcal{V}$. Then $u=a+v$ for some $a \in \mathcal{A}$ and $v \in \mathcal{V}$. Set $\mathcal{U}^{\prime}=\{a\}$. Then $\mathcal{U}^{\prime} \leq \mathcal{A}$ and $\mathcal{U} \leq \mathcal{U}^{\prime}+\mathcal{V}$, as required.

Following Theorem 3.9 every integral $\mu$ on $P$ is already determined by its values on the subcone $C=U$ of singleton sets in $P$, i.e. by a continuous linear functional $\mu_{0}$ in the usual dual $E^{\prime}$ of the locally convex vector space $E$. Using the result of Theorem 3.11 we infer that $\mu(\mathcal{A})=\sup \left\{\mu_{0}(a) \mid a \in \mathcal{A}\right\}$ for every $\mathcal{A} \in P$. This describes a one-to-one correspondence between $E^{\prime}$ and the integrals on $P$.

(c) In Example 1.2(c) we choose for $L$ the subcone of $P$ of all elements $l \oplus v$, where $l$ is a lower semicontinuous function in $F$ and $v \in V \cup\{\{0\}\}$, and for $U$ the cone of all elements $u \oplus\{0\}$, where $u$ is a real-valued upper semicontinuous function in $F$ with compact support. Note that those functions $u$ are bounded above. Then $L$ is a full cone as required, and $-U \subset L$. Following Remark 3.12(a), condition (L) needs not to be verified, whereas condition $\left(\mathrm{U}^{\prime}\right)$ may be easily checked:

Let $0+\{0\} \leq(f \oplus w)+\left(l \oplus w^{\prime}\right)=(f+l) \oplus\left(w+w^{\prime}\right)$ for $f \in F, l \in L$ and $w, w^{\prime} \in V \cup\{\{0\}\}$. Then $0 \leq(f+l)+\left(h+h^{\prime}\right)$, i.e. $-\left(l+h^{\prime}\right) \leq f+h$ for some lower semicontinuous functions $h \in w$ and $h^{\prime} \in w^{\prime}$. But the function $f+h \in F$ is (by the definition of $F$ ) bounded below by some $-\kappa \chi_{Y}$, where $\kappa>0$ and $\chi_{Y}$ denotes the (lower semicontinuous) characteristic function of a relatively compact open subset $Y$ of $X$. We set $u^{\prime}=\sup \left\{-\left(l+h^{\prime}\right),-\kappa \chi_{Y}\right\}$. Then $u^{\prime}$ is upper semicontinuous, bounded below and with compact support, thus $u^{\prime} \oplus\{0\} \in U$. The above shows that $u^{\prime} \leq f+h$ and $0 \leq u^{\prime}+\left(l+h^{\prime}\right)$, hence $u^{\prime} \oplus\{0\} \leq f \oplus w$ and $0+\{0\} \leq\left(u^{\prime} \oplus\{0\}\right)+\left(l \oplus w^{\prime}\right)$. The condition in $\left(\mathrm{U}^{\prime}\right)$ therefore holds with any choice of a neighborhood $0 \oplus v$.

It is also immediate that the integration cone $(P, L, U)$ is simplicial, as $U \cap L$ consists of the elements $c \oplus\{0\} \in P$, where $c$ is a continuous function on $X$ with compact support, and the condition in Definition 3.7 states a well-known fact about semicontinuous functions on a locally compact Hausdorff space. Likewise, $(P, L, U)$ is seen to be almost full.

Every continuous linear functional $\mu$ on $F$ may be extended to $P$ if we set $\mu(0 \oplus v)=\sup \{\mu(h) \mid h \in v\}$ for every $v \in V \cup\{\{0\}\}$. Considering this and 
the result from Theorem 3.11, our criterion in Theorem 3.6 for integrability of a function in $F$ will read as follows: A function $f \in F$ is $\mu$-integrable if and only if

$$
\begin{aligned}
\mu(f) & =\sup \left\{\mu(u) \mid u \leq f, \quad u \in U_{0}\right\} \\
& =\inf \left\{\mu(l) \mid f \leq l, \quad l \in L_{0}\right\},
\end{aligned}
$$

where $L_{0} \subset F$ denotes the subcone of $\overline{\mathbb{R}}$-valued lower semicontinuous functions, $U_{0} \subset F$ the real-valued upper semicontinuous functions with compact support. Following Theorem 3.11, for functions in $u \in U_{0}$ and $l \in L_{0}$ the integral is in turn determined by

$$
\begin{aligned}
\mu(l) & =\sup \left\{\mu(c) \mid c \leq l, c \in C_{0}\right\} \quad \text { and } \\
\mu(u) & =\inf \left\{\mu(c) \mid u \leq c, c \in C_{0}\right\},
\end{aligned}
$$

where $C_{0}=L_{0} \cap U_{0}$ denotes the continuous functions with compact support on $X$. The integrals of our theory, restricted to the subcone $F$ of $P$, therefore are the positive Radon measures on the locally compact space $X$, and our notion of integrability coincides with the usual one (cf. [5], IV.4, Théorème 3), except for the fact that we allow our integrals to take the value $+\infty$. Theorem 3.9 states that every continuous (positive) linear functional on the subspace $C_{0}$ of continuous functions with compact support permits a unique extension to a positive Radon measure on $X$, which is the result of the Riesz Representation Theorem.

Again we remark that this example simplifies considerably if we consider only compact spaces $X$.

(d) In Example 1.2(d) we choose $L=P$ and for $U$ the subcone of $P$ of all universally continuous elements (cf. [4], 4.1). Condition $\left(\mathrm{U}^{\prime \prime}\right)$ in 3.12(c) follows from the Riesz decomposition property and the fact that $U$ is specifically solid ([4], 4.1.2(a)). As $U \subset L$, the integration cone $(P, L, U)$ is also simplicial and almost full. The integrals of our definition coincide with the usual $\mathrm{H}$-integrals on the $\mathrm{H}$-cone $P$, if $P$ contains sufficiently many universally continuous elements, for example, if $P$ is a standard $H$-cone $([4], 4.2)$.

(e) Let $P=\{f \in C([0,1]) \mid f(0) \geq 0\}$ be endowed with the pointwise algebraic operations and order, and let $V \subset P$ consist of the positive constants. Let the full subcone $L$ consist of all functions $l$ in $P$ such that $l$ is concave and (right-hand) differentiable in 0 . Set $U=-L \cap P$, i.e. the functions in $U$ are convex, vanish and are differentiable in 0 . We shall check condition $\left(\mathrm{U}^{\prime}\right)$ from 3.12(a): Let $a \in P, l \in L$ such that $0 \leq a+l$, and let $v \in V$, i.e. $v=\varepsilon>0$. As $a$ is continuous in 0 , and $a(0) \geq 0$, there is $\delta>0$ such that $a(x) \geq-\varepsilon$ for all $x \in[0, \delta]$. Let $M<0$ be a lower bound for the function $a$ on $[0,1]$, and set $u^{\prime}(x)=\max \{(M / \delta) x,-l(x)\}$. Clearly $u^{\prime} \in U$, and $u^{\prime}(x) \leq a(x)+\varepsilon, \quad 0 \leq u^{\prime}(x)+l(x)$ for all $x \in[0,1]$, as required. Thus, $(P, L, U)$ is an integration cone. It is seen to be simplicial, but it is not almost full: For the function $a(x)=-\sqrt{x}$ in $P$, for example, there is no $u^{\prime} \in U$ such that $u^{\prime} \leq a$. The integrals on $P$ are just the positive multiples of the point evaluation in 1 , and every function in $P$ is seen to be integrable.

(f) This example will model complex Choquet theory as presented in [8]: Let $X$ be a compact space, and let $M$ be a subspace of $C_{\mathbb{C}}(X)$, the space of complexvalued continuous functions on $X$. By $\Gamma$ let us denote the unit circle in $\mathbb{C}$. Let $P=C(X \times \Gamma)$ be the space of all real-valued continuous functions on $X \times \Gamma$ with the pointwise operations and order and the positive constants as the neighborhood system $V \subset P$. A function $\rho \in P$ is said to be $M$-superharmonic if, firstly, its positively homogeneous extension on $X \times \mathbb{C}$ (that is $\rho(x, \lambda \gamma)=\lambda \rho(x, \gamma)$ for $\lambda \geq 0$ ) 
for every fixed $x \in X$ is sublinear as a functional on $\mathbb{C}$ and, secondly, if for all $x \in X$ and every complex measure $\mu=\chi|\mu|$ of norm at most 1 on $X$ such that $\mu(x)=m(x)$ for all $m \in M$, we have $|\mu|\left(\rho_{\gamma \chi}\right) \leq \rho(x, \gamma)$ for every $\gamma \in \Gamma$, where $\rho_{\gamma \chi}(x)=\rho(x, \gamma \chi(x))$. For our theory of integration cones we choose for $L$ and $-U$ the subcone of all such $M$-superharmonic functions in $P$. Following Remark 3.12(b), conditions (U) and (L) need not to be checked in this case, and the integration cone $(P, L, U)$ is almost full. Let us try to understand the integrals on $P: C_{\mathbb{C}}(X)$ may be embedded into $C(X \times \Gamma)$, if we set $\tilde{f}(x, \gamma)=\Re(\gamma f(x))$ for a function $f \in C_{\mathbb{C}}(X)$. Thus, the integrals $\mu$ on $P$ of our theory, when restricted to $C_{\mathbb{C}}(X)$, define a real-linear functional $\mu_{0}$ that may be complexified in the usual way, setting $\mu_{\mathbb{C}}(f)=\mu_{0}(f)-i \mu_{0}(i f)$. In this way, $\mu$ induces a complex Borel measure $\mu_{\mathbb{C}}=$ $\chi\left|\mu_{\mathbb{C}}\right|$ on $X$. It may be checked using Theorem 3.11 that for every $l \in L$ we have $\mu(l)=\sup \left\{\Re\left(\mu_{\mathbb{C}}(f)\right) \mid \tilde{f} \leq l, f \in C_{\mathbb{C}}(X)\right\}=\left|\mu_{\mathbb{C}}\right|\left(l_{\chi}\right)$. It will follow from this and our upcoming results that the measure $\mu_{\mathbb{C}}$ is supported in the sense of Choquet theory by the Choquet boundary of the subspace $M$.

\section{Convergence theorems}

In order to prepare a Lebesgue type convergence theorem for integrable elements in an integration cone $(P, L, U)$ we need to introduce a subset of special integrals that correspond to the point evaluations in classical integration theory. We shall use the notations of the previous sections, in particular the symbol $\equiv$ to denote equivalence of integrals (cf. the remark following Theorem 3.6).

Definition 4.1. Let $(P, L, U)$ be an integration cone. For a neighborhood $v \in V$ we define the integral boundary relative to $v$ to be the set $\Delta v$ of all integrals $\delta$ on $P$ such that $\delta(v)<+\infty$, satisfying the following property: If for integrals $\mu_{1}, \mu_{2}$ on $P$

$$
\delta(v)=\left(\mu_{1}+\mu_{2}\right)(v) \quad \text { and } \quad \delta(u) \leq\left(\mu_{1}+\mu_{2}\right)(u) \quad \text { for all } \quad u \in U,
$$

then there are $\lambda_{1}, \lambda_{2} \geq 0$ such that $\mu_{1} \equiv \lambda_{1} \delta$ and $\mu_{2} \equiv \lambda_{2} \delta$.

Note that for integrals $\delta, \mu_{1}$ and $\mu_{2}$ the condition that $\delta(u) \leq\left(\mu_{1}+\mu_{2}\right)(u)$ holds for all $u \in U$ implies already that

$$
\delta(a)=\check{\delta}(a) \leq\left(\mu_{1}+\mu_{2}\right)^{\vee}(a) \leq\left(\mu_{1}+\mu_{2}\right)(a)
$$

holds for all $\delta$-integrable elements $a \in P$. If $\delta(v)=0$ for an integral $\delta$ on $P$ and $v \in V$, then $\delta(u)=0$ holds for all $u \in U$, as the elements of $U$ are supposed to be bounded. Consequently $\delta \equiv 0$, and $\delta \in \Delta v$ for all $v \in V$. For $0 \not \equiv \delta \in \Delta v$, the constants $\lambda_{1}$ and $\lambda_{2}$ in Definition 4.1 are obviously given by $\lambda_{1}=\mu_{1}(v) / \delta(v)$ and $\lambda_{2}=\mu_{2}(v) / \delta(v)$, whence $\lambda_{1}+\lambda_{2}=1$.

Before we state our main theorem, let us identify the subsets $\Delta v$ of integrals on $P$ in our preceding examples:

Examples 4.2. (a) In Example 3.13(a), for a neighborhood $v \in V$, i.e. an $\overline{\mathbb{R}}$ valued strictly positive lower semicontinuous concave function on $X, \Delta v$ consists of the positive multiples of evaluations in those extreme points $x$ of $X$, where $v(x)<+\infty$.

(b) In 3.13(b) the integrals are determined by the dual $E^{\prime}$ of $E$. For a neighborhood $\mathcal{V} \in V$ the integral-boundary relative to $\mathcal{V}$ consists of those integrals on $P$ that are induced by positive multiples of the extreme points of the usual polar of $\mathcal{V}$ in $E^{\prime}$. 
(c) In 3.13(c), for a given neighborhood $v \in V$, the elements of $\Delta v$ are positive multiples of the evaluations in those points of $X$, where the functions in $v$ are uniformly bounded above, i.e. those points $x \in X$ such that $f(x) \leq M_{x}$ for all $f \in v$ and some $M_{x}<+\infty$.

(d) We shall see in the following section that in 3.13(d) the integral boundary consists of certain infimum homomorphisms on $P$.

(e) In 3.13(e) the positive multiples of the point evaluation in 1 are the only elements of $\Delta v$.

(f) It is easy to observe that in 3.13(f) the subcone $C=U \cap L$ consists only of functions in $C_{\mathbb{C}}(X)$, more precisely: the elements of the closure of the subspace $M$. The definition of $M$-superharmonic functions that form the subcones $-U=L$ yields that $\Delta v$ consists of the positive multiples of the extreme points of the polar of $v$ in the dual of $M$, hence corresponds to the usual Choquet boundary of $M$.

We are ready to prove our main convergence result. For a neighborhood $v \in V$ we shall say that a subset $A$ of $P$ is uniformly $v$-dominated if there is $\rho \geq 0$ such that $a \leq \rho v$ for all $a \in A$.

Theorem 4.3. Let $\mu$ be an integral on the integration cone $(P, L, U)$. For a neighborhood $v \in V$ let $\left(a_{n}\right)_{n \in \mathbb{N}}$ be a uniformly $v$-dominated sequence of $\mu$-integrable elements in $P$. If

$$
\lim \sup _{n \in \mathbb{N}} \delta\left(a_{n}\right) \leq \delta(v) \quad \text { for all } \quad \delta \in \Delta v
$$

then

$$
\lim \sup _{n \in \mathbb{N}} \mu\left(a_{n}\right) \leq \mu(v) .
$$

Proof. Our argument is modelled after the classical Bishop-De Leeuw theorem (cf. [1], I.4.10 or [8], 2.5): Let $v \in V$ and $a_{n} \in P$ be as in the statement, and let $\mu$ be an integral on $P$. If $\mu(v)=+\infty$, there is nothing to prove. If $\mu(v)=0$, then $\mu\left(a_{n}\right)=0$ for all $n \in \mathbb{N}$ as well because all the elements $a_{n}$ were supposed to be bounded by a multiple of $v$. Obviously our statement holds in this case. Otherwise, we may assume that $\mu(v)=1$.

By $K=v_{U}^{\circ}$ we denote the polar of $v$ in the dual $U^{*}$ of the locally convex cone $U . K$ is known to be convex and compact in the topology $w\left(U^{*}, U\right)([7]$, II.2.4). As the elements $a_{n} \in Q$ are supposed to be $\mu$-integrable, by Theorem 3.6 there is a sequence $\left(u_{n}\right)_{n \in \mathbb{N}}$ in $U$ such that $u_{n} \leq a_{n}+(1 / n) v$ and $\mu\left(u_{n}\right) \geq \mu\left(a_{n}\right)-1 / n$. Next we define a mapping $\Phi: K \rightarrow \mathbb{R}^{\mathbb{N}}$ by $\Phi(\nu)=\left(\nu\left(u_{n}\right)\right)_{n \in \mathbb{N}}$. Because $\Phi$ is continuous and affine, its range $Y=\Phi(K)$ is a compact convex subset of the metrizable space $\mathbb{R}^{\mathbb{N}}$. Now consider the sup-stable point-separating cone of continuous functions on $Y$, generated by the constants and the canonical projections $p_{n}: \mathbb{R}^{\mathbb{N}} \rightarrow \mathbb{R}$. By $\Delta Y$ we denote its classical Choquet boundary in $Y$. Our aim is to show that for every point $y \in \Delta Y$, the set $Z=\Phi^{-1}(y)$ contains the restriction on $U$ of an element $\delta \in \Delta v$. We argue as follows:

Clearly $Z$ is a closed convex subset of $K$ and a face, as the elements of $\Delta Y$ are also extreme points of $Y$. We consider the collection $\mathcal{F}$ of all non-empty compact convex faces $F$ in $Z$ with the property that for $\nu \in F$ and $\nu^{\prime} \in K, \quad \nu(u) \leq \nu^{\prime}(u)$ for all $u \in U$ implies that $\nu^{\prime} \in F$. As $y \in \Delta Y$, we observe that $Z$ itself fulfils this condition, hence $\mathcal{F} \neq \emptyset$ : If for $\nu \in Z$, and $\nu^{\prime} \in K, \quad \nu(u) \leq \nu^{\prime}(u)$ holds for all $u \in U$, then $p_{n}\left(\Phi\left(\nu^{\prime}\right)\right)=\nu^{\prime}\left(u_{n}\right) \geq \nu\left(u_{n}\right)=p_{n}(\Phi(\nu))=p_{n}(y)$ for all $n \in \mathbb{N}$. Thus 
$\nu^{\prime} \in \Phi^{-1}(y)=Z$ by the maximality of $y$. Furthermore, $\mathcal{F}$ is ordered inductively by inclusion and, following Zorn's Lemma, contains a minimal element $F_{0}$. Now we shall demonstrate that $F_{0}$ is in fact a singleton set: For an element $u_{0} \in U$ choose $\nu_{0} \in F_{0}$ such that $\nu_{0}\left(u_{0}\right)=\max \left\{\nu\left(u_{0}\right) \mid \nu \in F_{0}\right\}$. Set $G_{0}=\left\{\nu \in F_{0} \mid\right.$ $\left.\nu\left(u_{0}\right)=\nu_{0}\left(u_{0}\right)\right\}$. It is straightforward to check that $G_{0} \in \mathcal{F}$, hence $G_{0}=F_{0}$ by the minimality of $F_{0}$, which shows that $F_{0}$ is a singleton set, indeed. As $F_{0}$ is also a face in $Z$, hence in $K$, its only element $\delta_{0}$ is seen to be even an extreme point of $K$.

Next we use Theorem 3.3 to find an integral $\delta \in v_{P}^{\circ}$ such that $\delta_{0}(u) \leq \delta(u)$ for all $u \in U$. But the latter shows that the restriction of the integral $\delta$ to $U$ is also contained in $F_{0}$, hence equals $\delta_{0}$, and $\delta$ is in fact an extension of $\delta_{0}$. We shall proceed to demonstrate that $\delta$ is even contained in $\Delta v:$ As $\delta \in v_{P}^{\circ}$, we know that $\delta(v) \leq 1<+\infty$. If $\delta(v)=0$, then $\delta \equiv 0 \in \Delta v$ (cf. the remark following Definition 4.1). Otherwise, let $\mu_{1}, \mu_{2}$ be integrals such that

$$
\delta(v)=\left(\mu_{1}+\mu_{2}\right)(v) \quad \text { and } \quad \delta(u) \leq\left(\mu_{1}+\mu_{2}\right)(u) \text { for all } u \in U .
$$

Set $\lambda_{1}=\mu_{1}(v) / \delta(v)$ and $\lambda_{2}=\mu_{2}(v) / \delta(v)$. If $\lambda_{1}=0$, then $\mu_{1}(u)=0$ for all $u \in U$, as the elements of $U$ were supposed to be bounded. Then $\delta(u) \leq \mu_{2}(u)$, hence even $\delta(u)=\mu_{2}(u)$ holds for all $u \in U$, as $\left\{\delta_{0}\right\}=F_{0}$. The condition in 4.1 is satisfied in this case. A similar argument applies if $\lambda_{2}=0$. Otherwise, denote by $\delta_{1}$, resp. $\delta_{2}$, the restriction of $\left(1 / \lambda_{1}\right) \mu_{1}$, resp. $\left(1 / \lambda_{2}\right) \mu_{2}$ to $U$. Then $\lambda_{1} \delta_{1}+\lambda_{2} \delta_{2} \in F_{0}$ by the defining property for the sets in $\mathcal{F}$, thus $\lambda_{1} \delta_{1}+\lambda_{2} \delta_{2}=\delta_{0}$, and $\delta_{0}=\delta_{1}=\delta_{2}$ by the above. This verifies that the integrals $\delta, \mu_{1}$ and $\mu_{2}$ are equivalent, as they coincide on $U$.

Summarizing, for every $y \in \Delta Y$ we have, with the integral $\delta \in \Delta v$ from above and its restriction $\delta_{0} \in \Phi^{-1}(y)$, for all projections $p_{n}$ on $\mathbb{R}^{\mathbb{N}}$ that

$$
p_{n}(y)=p_{n}\left(\Phi\left(\delta_{0}\right)\right)=\delta_{0}\left(u_{n}\right)=\delta\left(u_{n}\right) \leq \delta\left(a_{n}+(1 / n) v\right) \leq \delta\left(a_{n}\right)+1 / n .
$$

Thus,

$$
\lim \sup _{n \in \mathbb{N}} p_{n}(y) \leq \lim \sup _{n \in \mathbb{N}} \delta\left(a_{n}\right) \leq \delta(v) \leq 1
$$

holds for all $y \in \Delta Y$.

Finally, as the restriction $\mu_{0}$ of $\mu$ to the subcone $U$ is contained in $K$, by Choquet's theorem for metrizable spaces (cf. [1], Theorem I.5.19) there is a probability measure $\psi$ on $Y$ representing the point $\Phi\left(\mu_{0}\right)$ for all projections $p_{n}$ and supported $\Delta Y$. The sequence $\left(p_{n}\right)_{n \in \mathbb{N}}$ is upper bounded on $Y$, since $\left(a_{n}\right)_{n \in \mathbb{N}}$ is upper bounded on $K$. Using Fatou's Lemma for the measure $\psi$ we conclude that

$$
\lim \sup _{n \in \mathbb{N}} \mu\left(a_{n}\right)=\lim \sup _{n \in \mathbb{N}} \mu_{0}\left(u_{n}\right)=\lim \sup _{n \in \mathbb{N}} \psi\left(p_{n}\right) \leq \psi\left(\lim \sup _{n \in \mathbb{N}} p_{n}\right) \leq 1=\mu(v) .
$$

Reviewing our examples we realize that for 3.13(a) and (f) Theorem 4.3 provides the known characterization of maximal (boundary) measures from Choquet theory. It allows detailed information about sets (Baire sets that contain the Choquet boundary) which support those measures (cf. [1], Corollary I.4.12 and [8], Proposition 4.5). In Example 3.13(b), Theorem 4.3 may be easily illustrated geometrically in simple cases (for example if $E=\mathbb{R}^{2}$ ), whereas in $3.13(\mathrm{c}$ ) our result leads to the usual convergence theorems of measure theory.

For a second convergence result for integrals we consider integration cones $(P, L, U)$, where the subcone $L$ contains suprema of dominated directed upward 
families of its elements, compatible with the algebraic operations; more precisely: we require that for every directed upward family $F \subset L$ that is dominated by an element of $L$ there exists the supremum $\bigvee F$ in $L$, and

$$
l+\bigvee F=\bigvee(l+F)
$$

holds for all $l \in L$. Using the terminology from [4], we shall say that an element $a \in P$ is universally continuous, if for every dominated directed upward family $F \subset L$ such that $a \leq \bigvee F$, for every $v \in V$ there is $l \in F$ such that $a \leq l+v$.

Using these notations we are ready to formulate our second convergence theorem:

Theorem 4.4. Let $(P, L, U)$ be an integration cone, where the subcone $L$ contains suprema of dominated directed upward families of its elements, compatible with the algebraic operations, and suppose that all elements of $U$ are universally continuous. If $a \leq \bigvee F$ for a dominated directed upward family $F \subset L$ and $a \in P$, then

$$
\mu(a) \leq \sup \{\mu(l) \mid l \in F\}
$$

holds for every integral $\mu$ on $P$ such that a is $\mu$-integrable.

Proof. Let $F, a$ and $\mu$ be as in the theorem. For $\varepsilon>0$ choose $v \in V$ such that $\mu(v) \leq \varepsilon / 3$. Following Theorem 3.6, there is $u \in U$ such that $u \leq a+v$ and $\mu(a) \leq \mu(u)+\varepsilon / 3$. By our assumption on $L$ we have $\bigvee(v+F)=v+\bigvee F \geq a+v \geq u$. And as the element $u \in U$ was supposed to be universally continuous, there is some $v+l \in v+F$ such that $u \leq(v+l)+v=l+2 v$. Summarizing, this yields

$$
\mu(a) \leq \mu(u)+\varepsilon / 3 \leq \mu(l)+\varepsilon \leq \sup \{\mu(l) \mid l \in F\}+\varepsilon .
$$

For our Examples 3.13 we observe that the assumptions of Theorem 4.4 hold for 3.13(a) to (d): This is clear for Example 3.13(a), as the pointwise supremum of a directed upward family of lower semicontinuous concave functions on $X$ is again lower semicontinuous and concave. This supremum is compatible with the algebraic operations. Also, because the functions in $U$ are upper semicontinuous, it follows from Dini's theorem that they are universally continuous in the sense of our definition.

In 3.13(b) for a directed upward family $F \subset L=P$ we have $\bigvee F=\overline{\bigcup_{\mathcal{A} \in F} \mathcal{A}}$, and

$$
\mathcal{B} \oplus \bigvee F=\overline{\mathcal{B}+\bigcup_{\mathcal{A} \in F} \mathcal{A}}=\overline{\bigcup_{\mathcal{A} \in F}(\mathcal{B}+\mathcal{A})}=\bigvee(\mathcal{B}+F)
$$

holds for any $\mathcal{B} \in P$. Also, the elements of $U$, i.e. the singleton sets in $P$ are universally continuous, as $\mathcal{U}=\{u\} \leq \bigvee F$ means $u \in\left(\bigcup_{\mathcal{A} \in F} \mathcal{A}\right)+\mathcal{V}$ for every $\mathcal{V} \in V$, hence $u \in \mathcal{A}+\mathcal{V}$ for some $\mathcal{A} \in F$.

In 3.13(c), for such a family $F$ of objects $l \oplus v$ in $L$ the supremum $\bigvee F$ is given by $l_{0} \oplus v_{0}$, where $l_{0}$ is the pointwise supremum of the functions $l$ and $v_{0}$ the union of the neighborhoods $v$. The functions in $u \in U_{0}$ are upper semicontinuous and have compact support. It follows from Dini's theorem that the elements $u \oplus\{0\} \in U$ are universally continuous in the sense of our definition.

In Example 3.13(d) the assumptions of Theorem 4.4 follow straight from the definition of an $\mathrm{H}$-cone and by our choice of the subcone $U$.

The statement of Theorem 4.4 is readily understood in all these examples. It is well-known (cf. [5], 4.4, Corollary 1) for integration theory on locally compact Hausdorff spaces (our Example 3.13(c)). 


\section{DiRECTED CONES}

It is immediate from our definition that any positive multiple of an integral is again an integral. Our Example 3.13(b), however, shows that this needs not to be the case for the sum of two integrals, i.e. the set of integrals does not necessarily form a subcone of $P^{*}$. This phenomenon will be remedied if we impose additional conditions on the subcones $U$ and $L$. We shall use the notations of the previous sections.

Definition 5.1. Let $(P, L, U)$ be an integration cone. The subcone $U$ is said to be up-directed if:

For all $l \in L, u_{1}, u_{2} \in U$ such that $u_{i} \leq l$, for $i=1,2$, and for every $v \in V$ there is $u \in U$ such that $u_{i} \leq u+v$ and $u \leq l+v$, for $i=1,2$.

Likewise, the subcone $L$ is said to be down-directed if:

For all $l_{1}, l_{2} \in L, u \in U$ such that $u \leq l_{i}$, for $i=1,2$, and for every $v \in V$ there is $l \in L$ such that $u \leq l \leq l_{i}+v$, for $i=1,2$.

Note that the slight asymmetry in the above conditions for $U$ and for $L$ results from the fact that $L$ other than $U$ is required to be a full cone. The condition for $U$ is automatically guaranteed, if in $P$, for elements $u_{1}, u_{2} \in U$, there exists the supremum $u=u_{1} \vee u_{2} \in U$. Likewise, if in $P$ for $l_{1}, l_{2} \in L$, there exists the infimum $l_{1} \wedge l_{2} \in L$, then the above condition for $L$ holds.

Theorem 5.2. Let $(P, L, U)$ be an integration cone. If $U$ is up-directed and if $L$ is down-directed, then the integrals on $P$ form a subcone of $P^{*}$.

Proof. As the set of integrals in $P^{*}$ is closed for multiplication by positive scalars, we only have to show that for integrals $\mu$ and $\nu$, the functional $(\mu+\nu)$ is again an integral. We shall use the criterion of Theorem 3.2:

For $l \in L$, let $\lambda_{1}<\mu(l), \lambda_{2}<\nu(l$,$) and for \varepsilon>0$ let $v \in V$ such that $(\mu+\nu)(v) \leq \varepsilon$. As $\mu$ and $\nu$ are integrals, there are $u_{i} \in U, i=1,2$, such that $u_{i} \leq l+v$ and $\lambda_{i} \leq \mu\left(u_{i}\right)$. Because $U$ is up-directed, there exists $u \in U$ such that $u_{i} \leq u+v$ and $u \leq(l+v)+v=l+2 v$. Thus

$$
\begin{aligned}
\lambda_{1}+\lambda_{2} & \leq(\mu+\nu)(u+v) \leq(\mu+\nu)(u)+\varepsilon \\
& \leq \sup \{(\mu+\nu)(u) \mid u \leq l+2 v, u \in U\}+\varepsilon,
\end{aligned}
$$

whence $\mu(l)+\nu(l)=(\mu+\nu)(l) \leq(\mu+\nu)^{\vee}(l)$, as $\varepsilon>0$ in the above inequality is arbitrary and the neighborhood system $V$ is directed downward (in the usual sense). The reverse inequality is obvious, and we infer that $(\mu+\nu)(l)=(\mu+\nu)^{\vee}(l)$ holds.

Similarly, for $u \in U$ and $\varepsilon>0$, choose $v \in V$ such that $(\mu+\nu)(v) \leq \varepsilon$. For $l_{1}, l_{2} \in L$ such that $u \leq l_{i}, i=1,2$, as $L$ is down-directed, there is $l \in L$ such that $u \leq l \leq l_{i}+v$. Thus

$$
(\mu+\nu)^{\wedge}(u) \leq(\mu+\nu)(l) \leq \mu\left(l_{1}+v\right)+\nu\left(l_{2}+v\right) \leq \mu\left(l_{1}\right)+\nu\left(l_{2}\right)+\varepsilon
$$

holds for all such $l_{1}, l_{2} \in L$ and $\varepsilon>0$, demonstrating that

$$
(\mu+\nu) \hat{\Upsilon}(u) \leq \hat{\mu}(u)+\hat{\nu}(u)=(\mu+\nu)(u) .
$$

The reverse inequality is again obvious, and by Theorem $3.2(\mu+\nu)$ is seen to be an integral. 
In order to characterize the elements of the integral boundary from Definition 4.1 as directional functionals we need to further strengthen the assumptions of directedness on the subcones $U$ and $L$.

Definition 5.3. Let $(P, L, U)$ be an integration cone. The subcone $U$ is said to be compatibly up-directed if:

For all $a \in P, l \in L, u_{1}, u_{2} \in U$ such that $u_{i}+a \leq l$, for $i=1,2$, and for every $v \in V$ there is $u \in U$ such that $u_{i} \leq u+v$, for $i=1,2$, and $u+a \leq l+v$.

Likewise, the subcone $L$ is said to be compatibly down-directed if:

For all $a \in P, \quad l_{1}, l_{2} \in L, \quad u \in U$ such that $u \leq l_{i}+a$, for $i=1,2$, and for every $v \in V$ there is $l \in L$ such that $l \leq l_{i}+v$, for $i=1,2$, and $u \leq l+a$.

Note that the above conditions for $U$ resp. $L$ are automatically guaranteed, if in $P$, for elements $u_{1}, u_{2} \in U$ resp. $l_{1}, l_{2} \in L$, there exists the supremum $u=u_{1} \vee u_{2} \in$ $U$ resp. the infimum $l_{1} \wedge l_{2} \in L$ and if those are compatible with the algebraic operations in $P$.

Lemma 5.4. If $U$ is compatibly up-directed, then for every integral $\mu$ on $P$ and elements $u_{1}, u_{2} \in U$ there are integrals $\mu_{1}, \mu_{2}$ on $P$ such that $\mu(a)=\left(\mu_{1}+\mu_{2}\right)(a)$ for all $\mu$-integrable elements $a \in P$ and

$$
\mu_{1}\left(u_{1}\right)+\mu_{2}\left(u_{2}\right)=\inf \left\{\mu(l) \mid u_{i} \leq l, i=1,2, \quad l \in L\right\} .
$$

Similarly, if $L$ is compatibly down-directed, then for every integral $\mu$ on $P$ and elements $l_{1}, l_{2} \in L$ there are integrals $\mu_{1}, \mu_{2}$ on $P$ such that $\mu(a)=\left(\mu_{1}+\mu_{2}\right)(a)$ for all $\mu$-integrable elements $a \in P$ and

$$
\mu_{1}\left(l_{1}\right)+\mu_{2}\left(l_{2}\right)=\inf _{v \in V} \sup \left\{\mu(u) \mid u \leq l_{i}+v, i=1,2, \quad u \in U\right\} .
$$

Proof. Because both statements and their proofs are similar, we shall prove only the second one: Let $\mu$ be an integral on $P$ and let $l_{1}, l_{2} \in L$. We denote by $I$ the subcone of $P$ of all $\mu$-integrable elements and consider the locally convex cone $Q=I \times I$ with the usual operations and order of a Cartesian product and the neighborhood system $V \times V$. On the subcone $Q_{0}=\left\{\lambda\left(l_{1}, l_{2}\right)+(a, a) \mid \lambda \geq 0, a \in I\right\}$ of $Q$ we define the functional $\Phi$ by

$$
\Phi\left(c_{1}, c_{2}\right)=\inf _{v \in V} \sup \left\{\mu(u) \mid u \leq c_{i}+v, i=1,2, \quad u \in U\right\} .
$$

First we observe that $\Phi\left(l_{1}, l_{2}\right)>-\infty$, as for $v \in V$ such that $\mu(v) \leq 1$ there is $\rho>0$ such that $0 \leq l_{i}+\rho v, i=1,2$. For every $v^{\prime} \in V$, by 5.3 (with $a=\rho v$ and $u=0)$ there is $l \in L$ such that $l \leq l_{i}+v^{\prime} / 2$ and $0 \leq l+\rho v$, whence $\mu(l) \geq-\rho$. Furthermore, because $\mu$ is an integral, we know that $\mu(l)=\check{\mu}(l)$, and there is $u \in U$ such that $u \leq l+v^{\prime} / 2$, i.e. $u \leq l_{i}+v^{\prime}$ and $\mu(u) \geq-\rho-1$. As $v^{\prime} \in V$ was arbitrary, this yields $\Phi\left(l_{1}, l_{2}\right) \geq-\rho-1$, indeed. Clearly $\Phi\left(l_{1}+a, l_{2}+a\right) \geq$ $\Phi\left(l_{1}, l_{2}\right)+\Phi(a, a)=\Phi\left(l_{1}, l_{2}\right)+\mu(a)$ holds for all $a \in I$. We obtain the converse inequality as follows: Let $\varepsilon>0$ and $v \in V$ such that $\mu(v) \leq \varepsilon$. For $u \in U$ such that $u \leq\left(l_{i}+a\right)+v / 2=\left(l_{i}+v / 2\right)+a, \quad i=1,2$, by 5.3 there is $l \in L$ such that $l \leq\left(l_{i}+v / 2\right)+v / 2=l_{i}+v$ and $u \leq(l+v / 2)+a \leq l+a+v$, whence $\mu(u) \leq \mu(l)+\mu(a)+\varepsilon$. This yields

$$
\begin{aligned}
\sup \{\mu(u) & \left.\mid u \leq l_{i}+a+v / 2, i=1,2, u \in U\right\} \\
& \leq \sup \left\{\mu(u) \mid u \leq l_{i}+v, i=1,2, u \in U\right\}+\mu(a)+\varepsilon .
\end{aligned}
$$


Consequently, because the last inequality holds for all such neighborhoods $v \in V$, taking the infimum over all $v \in V$ on both sides, we obtain

$$
\Phi\left(l_{1}+a, l_{2}+a\right) \leq \Phi\left(l_{1}, l_{2}\right)+\mu(a)+\varepsilon
$$

and even

$$
\Phi\left(l_{1}+a, l_{2}+a\right) \leq \Phi\left(l_{1}, l_{2}\right)+\mu(a),
$$

as $\varepsilon>0$ was arbitrary. As obviously $\Phi\left(\lambda c_{1}, \lambda c_{2}\right)=\lambda \Phi\left(c_{1}, c_{2}\right)$ holds for all $\left(c_{1}, c_{2}\right) \in$ $Q_{0}$ and $\lambda \geq 0$, we infer that $\Phi$ is in fact a linear functional on $Q_{0}$. It is also monotone and continuous, as $\Phi(v, v)=\mu(v) \leq 1$ for some neighborhood $v \in V$. By the Extension Theorem II.2.9 from [7] $\Phi$ can be extended into a continuous linear functional on all of $Q$, which we shall also denote by $\Phi$. Now we define linear functionals $\nu_{i}, i=1,2$, on $I$ by $\nu_{1}(a)=\Phi(a, 0)$ and $\nu_{2}(a)=\Phi(0, a)$. Then $\nu_{1}(a)+\nu_{2}(a)=\Phi(a, a)=\mu(a)$ holds for all $a \in I$. The functionals $\nu_{1}$ and $\nu_{2}$ are seen to be continuous and may be extended to all of $P$. By Theorem 3.3 there are integrals $\mu_{1}, \mu_{2}$ on $P$ such that

$$
\mu_{i}(l) \leq \nu_{i}(l) \quad \text { and } \quad \mu_{i}(u) \geq \nu_{i}(u)
$$

for all $l \in L, u \in U$ and $i=1,2$. Consequently we have

$$
\left(\mu_{1}+\mu_{2}\right)(l) \leq \mu(l) \quad \text { and } \quad\left(\mu_{1}+\mu_{2}\right)(u) \geq \mu(u)
$$

for all $l \in L$ and $u \in U$. Because $\mu$ is an integral, the latter inequalities imply that even

$$
\mu(a)=\check{\mu}(a) \leq\left(\mu_{1}+\mu_{2}\right)^{\vee}(a) \leq\left(\mu_{1}+\mu_{2}\right)(a) \leq\left(\mu_{1}+\mu_{2}\right)^{\wedge}(a) \leq \hat{\mu}(a)=\mu(a),
$$

i.e. $\mu(a)=\left(\mu_{1}+\mu_{2}\right)(a)$, holds for all $a \in I$. Furthermore, we obtain

$$
\mu_{1}\left(l_{1}\right)+\mu_{2}\left(l_{2}\right) \leq \nu_{1}\left(l_{1}\right)+\nu_{2}\left(l_{2}\right)=\Phi\left(l_{1}, l_{2}\right) .
$$

On the other hand we realize that

$$
\begin{aligned}
\Phi\left(l_{1}, l_{2}\right)= & \inf _{v \in V} \sup \left\{\mu(u) \mid u \leq l_{i}+v, i=1,2, \quad u \in U\right\} \\
= & \inf _{v \in V} \sup \left\{\left(\mu_{1}+\mu_{2}\right)(u) \mid u \leq l_{i}+v, i=1,2, \quad u \in U\right\} \\
\leq & \inf _{v \in V} \sup \left\{\mu_{1}(u) \mid u \leq l_{i}+v, i=1,2, \quad u \in U\right\} \\
& \quad+\inf _{v \in V} \sup \left\{\mu_{2}(u) \mid u \leq l_{i}+v, i=1,2, \quad u \in U\right\} \\
\leq & \mu_{1}\left(l_{1}\right)+\mu_{2}\left(l_{2}\right) .
\end{aligned}
$$

Together with the above, this yields $\mu_{1}\left(l_{1}\right)+\mu_{2}\left(l_{2}\right)=\Phi\left(l_{1}, l_{2}\right)$ and completes our proof.

Definition 5.5. An integral $\mu$ on $P$ is said to be up-directional on $U$ if

$$
\mu\left(u_{1}\right) \vee \mu\left(u_{2}\right)=\inf \left\{\mu(l) \mid u_{i} \leq l, i=1,2, \quad l \in L\right\}
$$

holds for all $u_{1}, u_{2} \in U$. Likewise, $\mu$ is said to be down-directional on $L$ if

$$
\mu\left(l_{1}\right) \wedge \mu\left(l_{2}\right)=\inf _{v \in V} \sup \left\{\mu(u) \mid u \leq l_{i}+v, i=1,2, \quad u \in U\right\}
$$

holds for all $l_{1}, l_{2} \in L$. 
Obviously, if $u_{1} \vee u_{2} \in U$ for $u_{1}, u_{2} \in U$, then we have $\mu\left(u_{1}\right) \vee \mu\left(u_{2}\right)=\mu\left(u_{1} \vee u_{2}\right)$ for an integral $\mu$ that is up-directional on $U$.

Likewise, $\mu\left(l_{1}\right) \wedge \mu\left(l_{2}\right)=\mu\left(l_{1} \wedge l_{2}\right)$ holds, if $\mu$ is down-directional on $L$ and if $l_{1} \wedge l_{2} \in L$ for $l_{1}, l_{2} \in L$.

Theorem 5.6. Let $(P, L, U)$ be an integration cone, and let $v \in V$. If $U$ is compatibly up-directed, then every $\delta \in \Delta v$ is up-directional on $U$. Similarly, if $L$ is compatibly down-directed, then every $\delta \in \Delta v$ is down-directional on $L$.

Proof. We shall give the proof for the second statement: Let $\delta \in \Delta v$ and suppose that $L$ is compatibly down-directed. For $\delta \equiv 0$ there is nothing to prove. Otherwise, for $l_{1}, l_{2} \in L$, let the integrals $\mu_{1}$ and $\mu_{2}$ be as in the preceding lemma. By the definition of $\Delta v$ we have $\mu_{i} \equiv \lambda_{i} \delta$ for $\lambda_{i}=\mu_{i}(v) / \delta(v), i=1,2$. This yields

$$
\begin{aligned}
\delta\left(l_{1}\right) \wedge \delta\left(l_{2}\right) & \leq \lambda_{1} \delta\left(l_{1}\right)+\lambda_{2} \delta\left(l_{2}\right)=\mu_{1}\left(l_{1}\right)+\mu_{2}\left(l_{2}\right) \\
& =\inf _{v \in V} \sup \left\{\delta(u) \mid u \leq l_{i}+v, i=1,2, u \in U\right\} .
\end{aligned}
$$

The reverse inequality, however, is obvious, showing that

$$
\delta\left(l_{1}\right) \wedge \delta\left(l_{2}\right)=\inf _{v \in V} \sup \left\{\delta(u) \mid u \leq l_{i}+v, i=1,2, \quad u \in U\right\}
$$

holds, indeed.

The converse of the preceding result, however, requires additional conditions:

Theorem 5.7. Let $(P, L, U)$ be an integration cone and let $v \in V$. If $-L \subset L+U$, then every integral $\delta$ that is up-directional on $U$ and finite for $v$ is contained in $\Delta v$. Similarly, if $-U \subset L+U$, then every integral $\delta$ on $P$ that is down-directional on $L$ and finite for $v$ is contained in $\Delta v$.

Proof. We shall prove the second statement: Suppose that $-U \subset L+U$. Let $\delta$ be an integral on $P$ that is down-directional on $L$, and let $v \in V$ such that $\delta(v)<+\infty$. We shall demonstrate that $\delta \in \Delta v:$ For $\delta(v)=0$ we have $0 \equiv \delta \in \Delta v$. Otherwise, let $\mu_{1}$ and $\mu_{2}$ be integrals as in Definition 4.1, i.e.

$$
\delta(v)=\left(\mu_{1}+\mu_{2}\right)(v) \quad \text { and } \quad \delta(u) \leq\left(\mu_{1}+\mu_{2}\right)(u) \text { for all } u \in U .
$$

This implies that $\delta(a)=\check{\delta}(a) \leq\left(\mu_{1}+\mu_{2}\right)^{\vee}(a) \leq\left(\mu_{1}+\mu_{2}\right)(a)$ holds for all $\delta$ integrable elements $a \in P$. As $-U \subset L+U$, the elements of $-U$ are $\delta$-integrable, and we have even $\delta(u)=\left(\mu_{1}+\mu_{2}\right)(u)$ for all $u \in U$. We may assume that $\delta(v)=1$ and set $\lambda_{1}=\mu_{1}(v)$ and $\lambda_{2}=\mu_{2}(v)$. For any element $l \in L$ such that $\delta(l) \geq 1$ we conclude that

$$
\begin{aligned}
1=\delta(v) \wedge \delta(l)= & \inf _{v^{\prime} \in V} \sup \left\{\delta(u) \mid u \leq v+v^{\prime}, u \leq l+v^{\prime}, u \in U\right\} \\
= & \inf _{v^{\prime} \in V} \sup \left\{\left(\mu_{1}+\mu_{2}\right)(u) \mid u \leq v+v^{\prime}, u \leq l+v^{\prime}, u \in U\right\} \\
\leq & \inf _{v^{\prime} \in V} \sup \left\{\mu_{1}(u) \mid u \leq v+v^{\prime}, u \leq l+v^{\prime}, u \in U\right\} \\
& \quad+\inf _{v^{\prime} \in V} \sup \left\{\mu_{2}(u) \mid u \leq v+v^{\prime}, u \leq l+v^{\prime}, u \in U\right\} \\
\leq & \left(\mu_{1}(v) \wedge \mu_{1}(l)\right)+\left(\mu_{2}(v) \wedge \mu_{2}(l)\right) \\
\leq & \left(\lambda_{1}+\lambda_{2}\right)=1 .
\end{aligned}
$$


This shows $\mu_{1}(l) \geq \lambda_{1}$ and $\mu_{2}(l) \geq \lambda_{2}$. Thus $\mu_{i}(l) \geq \lambda_{i} \delta(l)$ holds for all $l \in L$ such that $\delta(l)>0, i=1,2$. On the other hand, if $\delta(l)=-\rho \leq 0$ for $l \in L$, then $\delta(l+(\rho+1) v)=1$, and by the above we have $\lambda_{i} \leq \mu_{i}(l+(\rho+1) v)=\mu_{i}(l)+(\rho+1) \lambda_{i}$, and $\mu_{i}(l) \geq-\rho \lambda_{i}=\lambda_{i} \delta(l)$ as well. Thus we have $\mu_{i}(l) \geq \lambda_{i} \delta(l)$ for all $l \in L$, and because $\mu_{1}$ and $\mu_{2}$ are integrals, even

$$
\mu_{i}(a)=\hat{\mu}_{i}(a) \geq \lambda_{i} \hat{\delta}(a) \geq \lambda_{i} \delta(a)
$$

for every element $a \in P$ that is $\mu_{i}$-integrable for $i=1,2$. For every $u \in U$, finally, this yields

$$
\delta(u)=\left(\mu_{1}+\mu_{2}\right)(u) \geq \lambda_{1} \delta(u)+\lambda_{2} \delta(u)=\delta(u),
$$

whence $\mu_{i}(u)=\lambda_{i} \delta(u)$, as $u$ is bounded and $\delta(u)$ is finite. But integrals that coincide on the subcone $U$ were seen to be equivalent. We have $\mu_{i} \equiv \lambda_{i} \delta$ for $i=1,2$, as claimed.

Remarks 5.8. If $-U=L$, as in some of our examples, then both conditions in 5.1 coincide and may be reformulated as follows:

For all $l_{0}, l_{1}, l_{2} \in L$ such that $0 \leq l_{0}+l_{i}$, for $i+1,2$, and for every $v \in V$ there is $l \in L$ such that $l \leq l_{i}+v$, for $i=1,2$, and $0 \leq l+l_{0}$.

Likewise, both conditions in 5.3 coincide in this case and read:

For all $a \in P, l_{0}, l_{1}, l_{2} \in L$ such that $a \leq l_{0}+l_{i}$, for $i+1,2$, and for every $v \in V$ there is $l \in L$ such that $l \leq l_{i}+v$, for $i=1,2$, and $a \leq l+l_{0}$.

Examples 5.9. Reviewing our Examples 3.13, we see that in all our integration cones $(P, L, U)$, with the exception of Example 3.13(b), the subcones $L$ contain the infima of any two of their elements: They are the pointwise infima of the involved functions in the Examples 3.13(a), (c), (e) and (f), whereas in 3.13(b) the infimum of two elements (closed convex sets) of $P$ exists only if their intersection is not empty. In 3.13(d) the infimum of two elements exists by the definition of an $\mathrm{H}$-cone. The infima in Examples 3.13(a), (c), (e) and (f) are also compatible with the algebraic operations, whence the respective subcones $L$ are compatibly down-directed in the sense of Definition 5.3.

Similarly, again with the exception of 3.13(b), the subcones $U$ in our examples contain suprema of any two of their elements, thus are up-directed. With the further exception of H-cones (3.13(d)), those suprema are compatible with the algebraic operations, whence the respective subcones $U$ are compatibly up-directed.

In the above cases Theorem 5.6 provides known characterizations for the extreme points of a compact convex set in Example 3.13(a), the point evaluations on a locally compact Hausdorff space in 3.13(c) and the Choquet boundary of a subspace of $C_{\mathbb{C}}(X)$ in $3.13(\mathrm{f})$.

\section{REFERENCES}

1. E. M. Alfsen, Compact convex sets and boundary integrals, Ergebnisse der Mathematik und ihrer Grenzgebiete, vol. 57, Springer Verlag, Heidelberg-Berlin-New York, 1971. MR 56:3615

2. B. Anger and C. Portenier, Radon integrals, an abstract approach to integration and Riesz representation through function cones, Progress in Mathematics, vol. 103, Birkhäuser, Boston, 1992. MR 92m:28001

3. H. Bauer, Maß- und Integrationstheorie, 2. Auflage, Walter de Gruyter, Berlin-New York, 1992. MR 93g:28001

4. N. Boboc, G. Bucur, and A. Cornea, Order and convexity in potential theory, Lecture Notes in Mathematics, vol. 853, Springer Verlag, Heidelberg-Berlin-New York, 1981. MR 82i:31011 
5. N. Bourbaki, Éléments de Mathématique, Fascicule XIII, Livre VI, Intégration, Hermann, 1965. MR 36:2763

6. B. Fuchssteiner and W. Lusky, Convex cones, vol. 56, North Holland Math. Studies, 1981. MR 83m: 46018

7. K. Keimel and W. Roth, Ordered cones and approximation, Lecture Notes in Mathematics, vol. 1517, Springer Verlag, Heidelberg-Berlin-New York, 1992. MR 93i:46017

8. W. Roth, A new concept for a Choquet ordering in $C_{\mathbb{C}}(X)$, J. London Math. Soc. (2) 34 (1986), 81-96. MR 87j:46024

Department of Mathematics, University of Bahrain, P.O. Box 32038, Bahrain 